\title{
Optical detection of the rapidly spinning white dwarf in V1460 Her
}

\author{
Ingrid Pelisoli ${ }^{\circledR},{ }^{1 \star}$ T. R. Marsh, ${ }^{1}$ R. P. Ashley, ${ }^{2}$ Pasi Hakala, ${ }^{3}$ A. Aungwerojwit, ${ }^{4}$ K. Burdge ${ }^{(0)}, 5$ \\ E. Breedt ${ }^{\circledR}, 6$ A. J. Brown ${ }^{\oplus},{ }^{7}$ K. Chanthorn, ${ }^{8}$ V. S. Dhillon ${ }^{\circledR},{ }^{7,9}$ M. J. Dyer ${ }^{\circledR},{ }^{7}$ M. J. Green ${ }^{\oplus},{ }^{10}$ P. Kerry, ${ }^{7}$ \\ S. P. Littlefair ${ }^{\circledR},{ }^{7}$ S. G. Parsons ${ }^{\circledR},{ }^{7}$ D. I. Sahman, ${ }^{7}$ J. F. Wild ${ }^{\circledR 7}$ and S. Yotthanathong ${ }^{8}$ \\ ${ }^{1}$ Department of Physics, University of Warwick, Gibbet Hill Road, Coventry CV4 7AL, UK \\ ${ }^{2}$ Isaac Newton Group of Telescopes, Apartado de Correos 321, E-38700 Santa Cruz de La Palma, Spain \\ ${ }^{3}$ Finnish Centre for Astronomy with ESO (FINCA), Quantum, University of Turku, FI-20014 Turku, Finland \\ ${ }^{4}$ Department of Physics, Faculty of Science, Naresuan University, Phitsanulok 65000, Thailand \\ ${ }^{5}$ Division of Physics, Mathematics and Astronomy, California Institute of Technology, Pasadena, CA 91125, USA \\ ${ }^{6}$ Institute of Astronomy, University of Cambridge, Madingley Road, Cambridge CB3 OHA, UK \\ ${ }^{7}$ Department of Physics and Astronomy, Hicks Building, The University of Sheffield, Sheffield S3 7RH, UK \\ ${ }^{8}$ National Astronomical Research Institute of Thailand, 260 Moo 4, T. Donkaew, A. Maerim, Chiangmai 50180, Thailand \\ ${ }^{9}$ Instituto de Astrofísica de Canarias, E-38205 La Laguna, Tenerife, Spain \\ ${ }^{10}$ Department of Astrophysics, School of Physics and Astronomy, Tel Aviv University, Tel Aviv 6997801, Israel
}

Accepted 2021 August 27. Received 2021 August 27; in original form 2021 August 13

\begin{abstract}
Accreting magnetic white dwarfs offer an opportunity to understand the interplay between spin-up and spin-down torques in binary systems. Monitoring of the white dwarf spin may reveal whether the white dwarf spin is currently in a state of nearequilibrium, or of unidirectional evolution towards longer or shorter periods, reflecting the recent history of the system and providing constraints for evolutionary models. This makes the monitoring of the spin history of magnetic white dwarfs of high interest. In this paper, we report the results of a campaign of follow-up optical photometry to detect and track the 39-s white dwarf spin pulses recently discovered in Hubble Space Telescope data of the cataclysmic variable V1460 Her. We find the spin pulsations to be present in the $g$-band photometry at a typical amplitude of 0.4 per cent. Under favourable observing conditions, the spin signal is detectable using 2-m class telescopes. We measured pulse-arrival times for all our observations, which allowed us to derive a precise ephemeris for the white dwarf spin. We have also derived an orbital modulation correction that can be applied to the measurements. With our limited baseline of just over $4 \mathrm{yr}$, we detect no evidence yet for spin-up or spin-down of the white dwarf, obtaining a lower limit of $|P / \dot{P}|>4 \times 10^{7} \mathrm{yr}$, which is already four to eight times longer than the time-scales measured in two other cataclysmic variable systems containing rapidly rotating white dwarfs, AE Aqr and AR Sco.
\end{abstract}

Key words: binaries: close-binaries: eclipsing-binaries: general-stars: cataclysmic variables.

\section{INTRODUCTION}

The spin rates of accreting white dwarfs in cataclysmic variable binary stars are determined by competing mechanisms, which add or extract angular momentum. On one hand, accreting material of high specific angular momentum drives the white dwarf to spin faster. The concentrated internal structures of white dwarfs, together with their shrinkage in response to increased mass, mean that only $0.1 \mathrm{M}_{\odot}$ of added matter is needed in principle to bring a white dwarf close to its few second maximal breakup spin rate (Livio \& Pringle 1998). However, there are no systems known to be very close to this limit, presumably because white dwarfs can also lose angular momentum in three ways. First, white dwarfs accreting hydrogenrich material undergo thermonuclear runaways once $1 \times 10^{-6}$ to $1 \times 10^{-4} \mathrm{M}_{\odot}$ of matter has accumulated, causing their envelopes to expand (Paczynski \& Zytkow 1978; Wolf et al. 2013). Coreenvelope coupling during these phases can be expected to slow the

^E-mail: ingrid.pelisoli@warwick.ac.uk white dwarfs' spin rates (Livio \& Pringle 1998). Second, magnetic white dwarfs couple through their fields to the accretion disc or to the binary companion, causing their spin rates to slow. In the limit of very strong fields their spins lock to the binary orbit, as seen in the polar class of accreting white dwarfs, also known as AM Her stars (Joss, Katz \& Rappaport 1979; Campbell 1983). A third possible mechanism is through tides, which are likely to be important for ultracompact orbits and during double white dwarf mergers (Fuller \& Lai 2012).

Magnetic white dwarfs are of particular interest because they reveal their spin periods through photometric variations induced by spots, which result from accretion rate asymmetries caused by their fields. This allows the measurement of extremely precise spin periods to reveal the competing effects of accretion versus magnetic drag on a year by year basis (Patterson 1984). This plays out in the intermediate polar (IP) class of system. IPs have spin periods of order minutes to tens of minutes (Patterson 1994). These are rates much slower than breakup, but faster than their binary periods, reflecting a quasi-equilibrium between spin-up and magnetic drag that is continuously adjusting to a fluctuating ac- 
cretion rate (Patterson 1984; Kennedy et al. 2016; Littlefield et al. 2020)

Not all systems exist in quasi-equilibrium. One of the first recognized cataclysmic variables, AE Aquarii, shows remarkable flaring behaviour that is thought to be driven by magnetically propelled material exiting the system (Wynn, King \& Horne 1997). AE Aqr was unique in this behaviour amongst the thousands of known systems until the recent discovery of LAMOST-J024048.51 + 195226.9 (Thorstensen 2020; Garnavich et al. 2021b). The white dwarf in AE Aqr has a spin period of $33 \mathrm{~s}$ (Patterson 1979) and is spinning down on a short $10^{7} \mathrm{yr}$ time-scale (de Jager et al. 1994), and it is thought that little or no accretion takes place. Instead the system is now in a spin-powered state, which powers not just the flares, but also a broad synchrotron spectrum extending to radio frequencies (Bookbinder \& Lamb 1987). AE Aqr is not in equilibrium, but is instead being observed in what appears to be a relatively brief evolutionary phase compared to the several billion year lifetimes of cataclysmic variables. A similar, possibly more advanced, state has been reached by the system AR Sco, which, like AE Aqr, is also a strong synchrotron source (Marsh et al. 2016). The white dwarf in AR Sco, whose spin period is $118 \mathrm{~s}$, is spinning down on a timescale of $5 \times 10^{6} \mathrm{yr}$ (Stiller et al. 2018; Gaibor et al. 2020), and in this case the system appears to be entirely detached (Marsh et al. 2016; Garnavich et al. 2021a), with no accretion and no evidence for propeller-induced flaring activity (Littlefield et al. 2017).

It is not yet clear how systems like AE Aqr and AR Sco achieved their current states. Although their magnetic field strengths have not been directly measured, the absence of accretion discs and the rates of spin-down have led to estimates ranging from 50 to 200 MG (Ikhsanov 1998; Katz 2017). White dwarfs with fields this high are hard to spin up to the short periods seen in AE Aqr and AR Sco, because very high accretion rates are needed to compress the magnetosphere down to a radius at which the Keplerian orbital period in the disc is this short. While there are signs of a high rate of accretion in AE Aqr's recent past (Schenker et al. 2002), there is no similar evidence in the case of AR Sco. Perhaps the field estimates are simply far too large (Lyutikov et al. 2020), but that would still leave an open question of why these two systems in particular have lost their discs.

A radically different hypothesis for AR Sco has been recently put forward by Schreiber et al. (2021). They proposed that the white dwarf in AR Sco only became magnetic as a result of a crystallizationand rotation-driven dynamo, similar to the mechanism thought to be at work in planets and low-mass stars. In their model, which was derived from an explanation for white dwarf magnetism initially put forward by Isern et al. (2017), the carbon-oxygen white dwarf in a post-common envelope binary with a main-sequence star is originally non-magnetic. As the system evolves towards shorter orbital periods, the white dwarf cools and the companion evolves towards a Roche lobe filling star. When that happens, the binary becomes a cataclysmic variable, and accretion spins up the white dwarf, whose core might be crystallizing. If that is the case, conditions for a dynamo - strong density stratification and convection - are met, generating a magnetic field. If the field is strong enough, the disc may be disrupted and connection with the secondary star field will provide a synchronizing torque on the white dwarf spin. Again, if the field is strong enough, the rapid transfer of spin angular momentum into the orbit may cause the binary to detach and mass transfer to cease. AR Sco might be an example of such a system. This provides a natural explanation for the high incidence of magnetism amongst cataclysmic variable stars (36 per cent, Pala et al. 2020), together with a near-total absence of magnetism amongst their progenitor systems (Liebert et al. 2005,
2015; Parsons et al. 2021). Importantly, it sidesteps the problem of spinning up a highly magnetic white dwarf.

Schreiber et al.'s (2021) hypothesis makes accreting white dwarfs of short spin period of very high interest, as they could be systems whose fields have only recently emerged, and they might now be in a state of rapid spin-down. Whether this is the case, or whether they are in fact in a state of quasi-equilibrium, possible for relatively weak fields at short spin periods, can only be established through observation.

One such short-period white dwarfs have been recently discovered in V1460 Her (Ashley et al. 2020). V1460 Her is an eclipsing cataclysmic variable with a 4.99-h orbital period and an overluminous K5-type donor star. It is the third fastest spinning white dwarf known amongst the cataclysmic variables, with a spin period of 38.875(5) s (Ashley et al. 2020). Amongst cataclysmic variable stars, only the white dwarfs in AE Aqr (33 s) and CTCV J2056-3014 (29.6s, Lopes de Oliveira et al. 2020) spin faster, although the X-ray binary HD 49798 contains a $P_{\text {spin }}=13 \mathrm{~s}$ compact component that might be a white dwarf (Israel et al. 1997; Mereghetti et al. 2011). An evolved companion is another unusual characteristic among CVs that is shared by V1460 Her and AE Aqr. Only $5 \pm 3$ per cent of CVs are found to have evolved companions (Pala et al. 2020), which is at odds with the theoretical prediction of 30 per cent (Schenker et al. 2002).

Since discovering the rapid pulsations betraying the presence of the white dwarf spin in V1460 Her's HST Ultraviolet (UV) data (Ashley et al. 2020), we have obtained multiple epochs of high-speed optical photometry in an effort to detect and monitor the spin. In this paper, we present the results of this campaign aimed at establishing the spin history of the magnetic white dwarf V1460 Her since its recent discovery.

\section{OBSERVATIONS}

Our follow-up observations of V1460 Her were carried out at the 2.0m Liverpool Telescope (LT), the 2.56-m Nordic Optical Telescope (NOT), the 4.2-m William Herschel Telescope (WHT), the Palomar 200-inch Telescope, and the 2.4-m Thai National Telescope (TNT). The details of our observing runs are shown in Table 1 .

At the LT, we used the optical wide-field camera IO:O with a $2 \times 2$ binning to reduce the readout time. Observations were taken with the Sloan $g$ filter. Given a rather slow readout time of $18 \mathrm{~s}$, we chose exposure times of 10-12 s to avoid too much loss of signal, even though the resulting cadence of 28.6-30.6s was sub-Nyquist for the spin signal in V1460 Her.

The WHT runs used the QHY CMOS detector at prime focus with a windowed configuration aimed at minimizing readout time. For the two initial runs in 2021 February, the Astronomik $B$ filter, which has a similar throughput to Sloan $g$, was used. For the following two runs, in 2021 May, the Sloan $g$ filter was used. The exposure time was set to $5 \mathrm{~s}$, which resulted on a cadence of $7.8 \mathrm{~s}$ accounting for the readout time of $2.8 \mathrm{~s}$.

At the TNT, the high-speed camera ULTRASPEC (Dhillon et al. 2014) was employed, with a $g$ filter and exposure time varying between 4.8 and $9.6 \mathrm{~s}$ depending on the observing conditions, with only 15-ms lost between exposures.

NOT observations were executed with ALFOSC, a multimode imager/spectrograph. We used a subwindow binned by a factor of 2 yielding a 2-s dead time. A $g$ filter was used, except for observations starting on 2020 May 30, which used a $U$ filter. The exposure time for both the $g$ - and $U$-band data was $3 \mathrm{~s}$. 
Table 1. Journal of observations.

\begin{tabular}{|c|c|c|c|c|c|}
\hline Run number & Telescope & Start date (TDB) & Duration (min) & Cadence (s) & Filter \\
\hline 1 & TNT & 2020-03-11 21:15:54.281 & 99.6 & 4.9 & $g$ \\
\hline 2 & TNT & 2020-03-13 21:06:52.664 & 53.5 & 4.8 & $g$ \\
\hline 3 & TNT & 2020-03-14 21:07:02.065 & 122.0 & 4.3 & $g$ \\
\hline 4 & TNT & 2020-03-15 20:59:57.109 & 76.7 & 7.3 & $g$ \\
\hline 5 & TNT & 2020-03-23 22:30:16.836 & 19.8 & 7.9 & $g$ \\
\hline 6 & NOT & 2020-05-02 02:58:03.805 & 63.7 & 5.0 & $g$ \\
\hline 7 & NOT & 2020-05-30 01:33:11.873 & 76.8 & 6.5 & $U$ \\
\hline 8 & NOT & 2020-07-30 23:33:21.091 & 63.4 & 5.0 & $g$ \\
\hline 9 & Palomar & 2020-07-31 04:29:04.918 & 33.6 & 1.0 & $g$ \\
\hline 10 & LT & 2021-01-29 05:36:56.940 & 49.5 & 30.6 & $g$ \\
\hline 11 & LT & 2021-02-14 05:24:35.987 & 49.5 & 30.6 & $g$ \\
\hline 12 & WHT & 2021-02-14 04:51:50.774 & 103.9 & 7.8 & $B$ \\
\hline 13 & WHT & 2021-02-17 03:13:28.577 & 153.2 & 7.8 & $B$ \\
\hline 14 & TNT & 2021-02-28 21:53:35.618 & 72.1 & 4.8 & $g$ \\
\hline 15 & TNT & 2021-03-01 21:57:58.907 & 71.8 & 5.0 & $g$ \\
\hline 16 & TNT & 2021-03-02 21:46:42.073 & 80.2 & 4.8 & $g$ \\
\hline 17 & LT & 2021-03-03 03:02:07.962 & 49.5 & 30.6 & $g$ \\
\hline 18 & TNT & 2021-03-09 21:27:09.409 & 94.1 & 5.4 & $g$ \\
\hline 19 & LT & 2021-03-18 04:06:46.081 & 49.1 & 28.6 & $g$ \\
\hline 20 & TNT & 2021-03-20 20:40:35.052 & 132.6 & 6.0 & $g$ \\
\hline 21 & TNT & 2021-03-20 20:40:35.052 & 132.6 & 6.0 & $g$ \\
\hline 22 & TNT & 2021-03-31 21:31:34.211 & 77.8 & 9.6 & $g$ \\
\hline 23 & TNT & 2021-04-01 21:22:14.809 & 81.8 & 9.6 & $g$ \\
\hline 24 & LT & 2021-04-02 05:09:16.121 & 49.3 & 28.6 & $g$ \\
\hline 25 & TNT & 2021-04-09 18:32:39.911 & 235.6 & 8.9 & $g$ \\
\hline 26 & TNT & 2021-04-10 20:31:04.867 & 116.0 & 8.9 & $g$ \\
\hline 27 & TNT & 2021-04-18 20:35:01.170 & 120.4 & 4.8 & $g$ \\
\hline 28 & TNT & 2021-04-19 18:52:00.371 & 58.2 & 4.8 & $g$ \\
\hline 29 & NOT & 2021-05-01 02:25:01.479 & 62.5 & 6.0 & $g$ \\
\hline 30 & TNT & 2021-05-02 20:18:49.762 & 121.3 & 4.8 & $g$ \\
\hline 31 & TNT & 2021-05-03 19:15:18.515 & 178.2 & 4.8 & $g$ \\
\hline 32 & TNT & 2021-05-04 20:18:16.380 & 21.0 & 9.6 & $g$ \\
\hline 33 & TNT & 2021-05-05 20:05:27.439 & 112.9 & 9.6 & $g$ \\
\hline 34 & WHT & 2021-05-19 22:05:18.250 & 440.0 & 7.8 & $g$ \\
\hline 35 & WHT & 2021-05-21 02:32:53.930 & 169.1 & 7.8 & $g$ \\
\hline
\end{tabular}

The Caltech HIgh-speed multicolour camERA (CHIMERA, Harding et al. 2016) provided data from the 200-inch Hale Telescope at Palomar observatory. The $g$ filter was used in the blue channel. In the red, the K-type star companion dominates the light, diluting the signal from the white dwarf. Therefore, only the blue channel data are used here. The CCD was operated using the $0.1-\mathrm{MHz}$ conventional amplifier with 1 -s exposures with $2 \times 2$ binning, and used frame transfer to effectively eliminate time lost due to reading out between exposures.

All our observations were bias subtracted and flat-field corrected. Observation times were corrected to Barycentric Julian Date (BJD) in the Barycentric Dynamical Time (TDB) reference system. For each image, differential aperture photometry was carried out. We have used a variable aperture size, set to scale with the seeing measured from a point spread function (PSF) fit, to accommodate variations in the atmospheric conditions. We chose as our main comparison Gaia EDR3 1382561212513336064, a nearby star with similar colour to V1460 Her and, in particular, showing low astrometric excess noise and Renormalised Unit Weight Error (RUWE) in Gaia, which are proxies for variability (Belokurov et al. 2020). For one of our TNT observations, using this comparison star yielded poor results. In this case, we have used instead a brighter comparison star, TYC 3068-855-1. Using this bright comparison is not possible for most of our observations, as it is often saturated. For the NOT runs, SDSS J162123.10 + 441241.6 was used as the comparison star.

\section{RESULTS}

\subsection{Detection of the pulsations}

We computed the amplitude spectrum for each separate run, following subtraction of a spline to remove orbital-related variations. Data taken while the white dwarf was eclipsed were masked. An example of this procedure is shown in Fig. 1, and the amplitude spectra of all runs are shown in Fig. 2. The spin pulsation signal was always detected in our WHT runs, and is clear in our CHIMERA run as well. It is also detected in the three $g$-band NOT observations, though with high uncertainty for one of them (due to bright Moon conditions), but not in the $U$-band run. For the TNT runs, the signal is detected in all but three runs, which were affected by detector pickup noise and/or poor observing conditions (clouds and/or bright Moon). In the case of the LT observations, detection is only marginally possible owing to the slow detector readout time along with the consequent sub-Nyquist sampling and loss of signal. We detected the signal with an uncertainty smaller than 30 per cent in only one of our five LT runs.

\subsection{Measurement of pulse times}

We measured a single time and uncertainty for each discrete run representing the time of peak flux $T_{0}$ by fitting a cosine with a period 

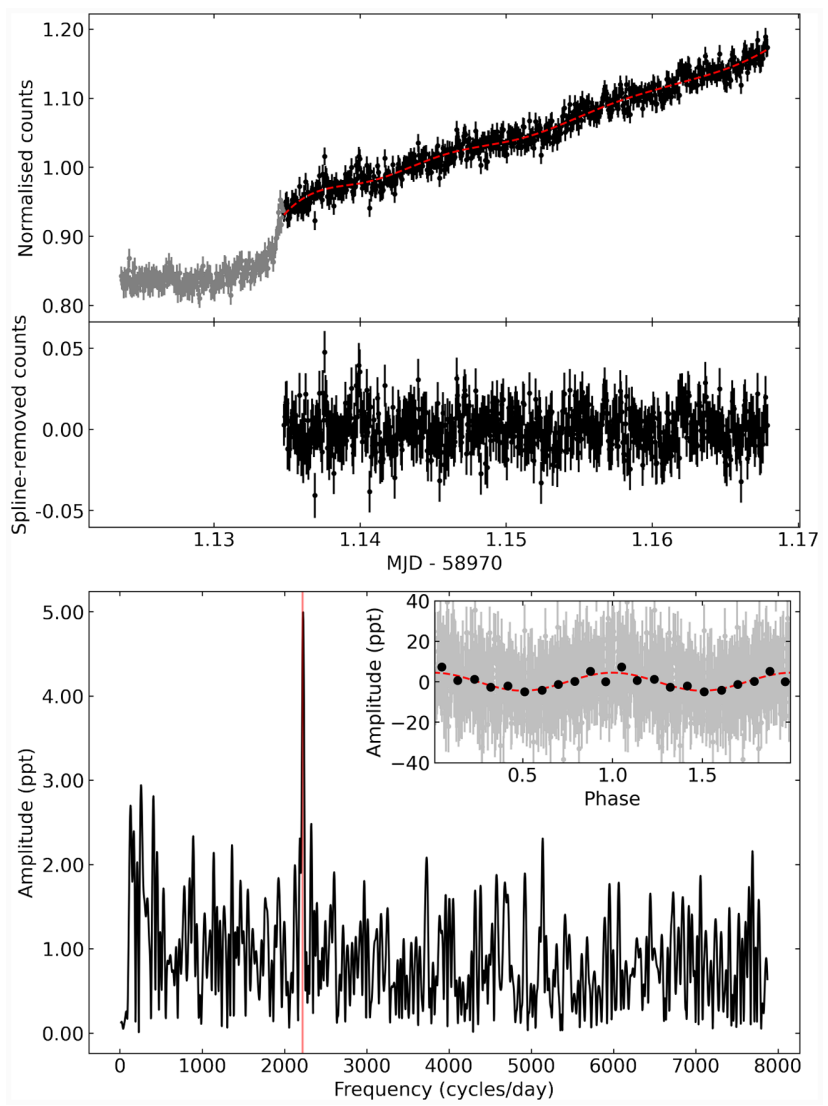

Figure 1. Top panel shows the original light curve, taken at the NOT on 2020 May 2. Data taken during an eclipse are shown in grey. Only the data shown in black were used for fitting a spline, shown as a red dashed line. The spline-subtracted data are shown in the middle panel. The amplitude spectrum of these data is shown in the bottom panel, with the spin period derived by Ashley et al. (2020) shown as a vertical red line. Amplitudes are shown in parts per thousand (ppt). The inset in the bottom panel shows the spline-subtracted data (in grey) folded on the spin period, with the cosine fit shown as a red dashed line. The black points show an average every 50 points to aid visualization.

fixed to Ashley et al.'s (2020) measurement (see Fig. 1 inset), but with an arbitrary zero-point in time. The fits were carried out to the spline-subtracted data described in Section 3.1. The zero-point times from such measurements are uncertain by arbitrary offsets of integer multiples of the period. However, any error in the period used for the fit will be amplified if offsets are chosen that move the selected time far from the data upon which it is based. Therefore, we selected values close to the mid-time of each data set in every case. Runs of a length significant compared to the 4.99-h orbital period were split into sub-sections in order to be sensitive to possible orbital modulation, which is expected if the periodic signal contains orbital side-bands as is often the case in intermediate polars (IPs; e.g. Kennedy et al. 2016; Patterson et al. 2020). Table 2 shows the measured times, along with cycle number counts whose derivation we detail in the next section. Uncertainties are given by the standard deviation after a thousand bootstrapping iterations, in which data points were sampled allowing for repeated values and then refitted.

\subsection{Fixing the cycle counts}

We calculated integer pulse cycle numbers for all data over a finely spaced grid covering two dimensions representing adopted spin period and spin phase offset, the two unknowns of the problem. We searched over a set of periods centred on the previously determined period of 38.875(5) s from Ashley et al. (2020), extending over an interval of $\pm 5 \sigma$ on either side, where $\sigma$ is Ashley et al.'s (2020) uncertainty estimate. The phase offset grid extended from 0 to 1, i.e. over an entire cycle. Any given period/phase offset pair within the grid leads to a set of integer cycle counts, which can then be used to fit a linear ephemeris to the data from which a $\chi^{2}$ value results. For any fixed period, it is important to optimize over the phase offset since it is not known in advance and it can result in cycle numbers flipping by plus or minus a cycle; hence, our use of a 2D search grid. Fig. 3 shows the resulting $\chi^{2}$ as a function of period, after selection of the minimum $\chi^{2}$ over all phase offsets for each period.

This is the stage at which one can tell if the observations are sufficient to fix the cycle counts uniquely. If they are not, there will be multiple aliases with similar minimum $\chi^{2}$ values. Any with comparable values of $\chi^{2}$ need to be regarded as potential candidate periods, with more data being required to distinguish between them. We find that the second deepest minimum has a $\chi^{2}$ more than 300 larger than our favoured period (see Fig. 3), and still $>100$ after scaling uncertainties to give reduced $\chi^{2}=1$. This is comfortably above the threshold difference of 10 found to yield reliable periods by Morales-Rueda et al. (2003) in their study of B-type hot subdwarf (sdB) orbital periods. ${ }^{1}$ This absence of any competing aliases shows that there is only one viable period and that the cycle counts are uniquely determined. The location of the best period within less than $2 \sigma$ of Ashley et al.'s (2020) favoured value is further support of the proposed solution.

In order to define the epoch of cycle number 0 , we performed a linear ephemeris fit for different $T_{0}$ values, separated by an integer number of cycles and spanning the time interval of our observations. We monitored the resulting covariance between $T_{0}$ and period in order to find the location of the minimum. We elected the value that minimized this covariance as $T_{0}$. This results in the following ephemeris for the time of maximum brightness:

$\mathrm{BMJD}(\mathrm{TDB})=58888.0183797(17)+0.0004498987920(12) \mathrm{E}$,

where $\mathrm{E}$ is the cycle number. The time-scale is TDB, corrected to the barycentre of the Solar system, expressed as a Modified Julian Day number (MJD = JD-400000.5). Uncertainties were determined from a thousand Monte Carlo runs of the linear ephemeris fit, resampling the derived times of maxima each time according to their uncertainties.

\subsection{Modulation with orbital period}

In order to identify whether the spin signal presents any orbital modulation, we have calculated the orbital phase for each of our measurements using the orbital ephemeris derived by Ashley et al. (2020). We have subtracted the calculated (C) cycle count from the observed $(\mathrm{O})$ value for each epoch to inspect the $\mathrm{O}-\mathrm{C}$ diagram for any hints of dependence on the orbital phase. The top panel of Fig. 4 shows the result for all our observing runs. The highly variable size of the error bars, which are strongly dependent on the instrument and observing conditions, makes it hard to achieve any conclusion regarding orbital modulation.

\footnotetext{
${ }^{1}$ The reasoning is that the probability of a period is dominated by the term $\exp \left(-\chi^{2} / 2\right)$; therefore a difference of more than 10 implies that the secondbest alias is at least $\exp (5) \simeq 150$ times less probable than the best.
} 


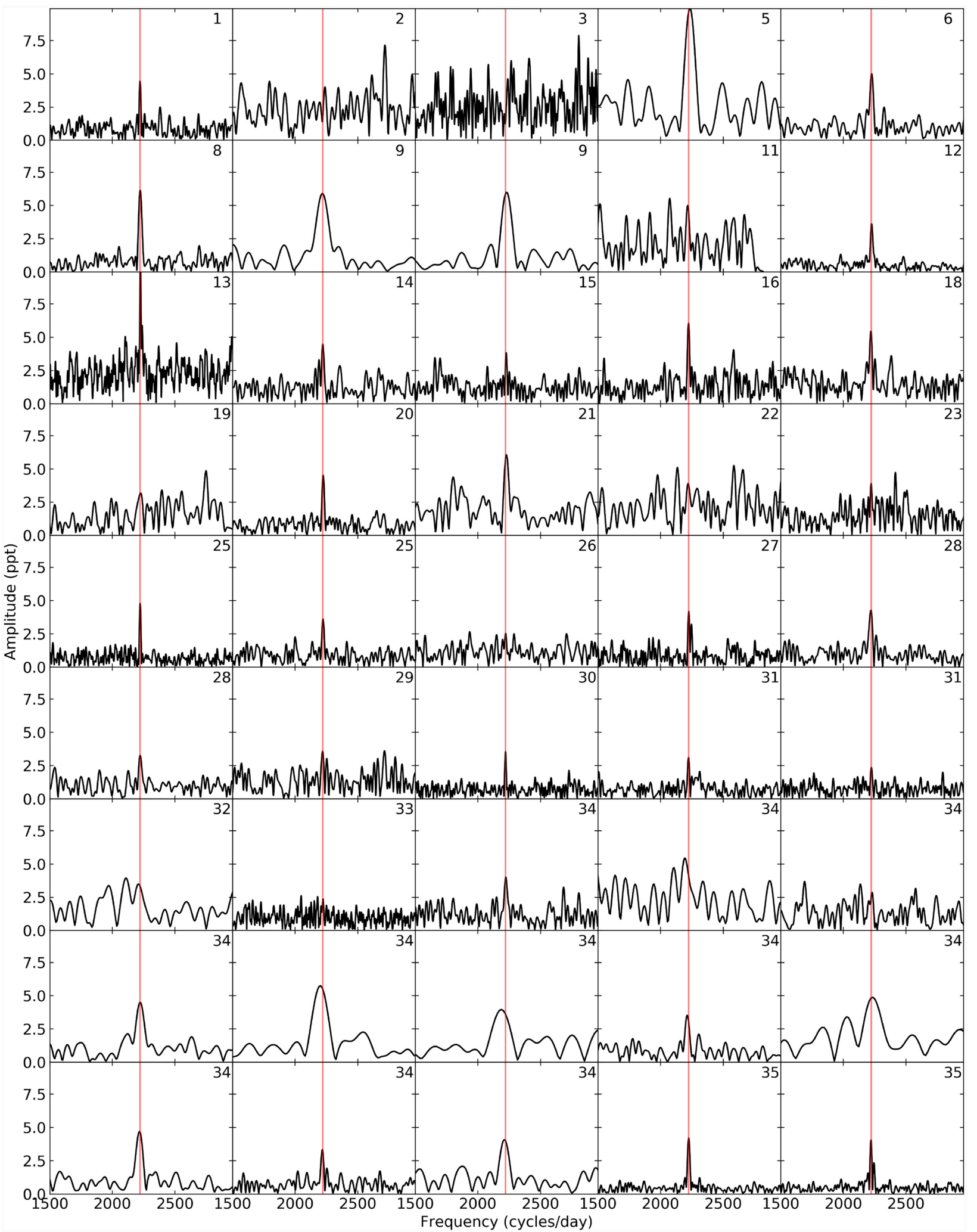

Figure 2. Amplitude spectra corresponding to the derived pulse times shown in Table 2. The run number is shown at the top right-hand corner, and the spin period obtained by Ashley et al. (2020) is marked by a vertical red line. As described in the text, long runs were split into smaller time intervals; therefore more than one panel is shown for a few runs, each panel corresponding to one of the time intervals. 
Table 2. Measured pulse times, with respective uncertainties, and the derived cycle count. As noted in the text, some runs were split into smaller time intervals and thus contributed to more than one $T_{0}$ measurement. Run number corresponds to that shown in Table 1, with the exception of the previously existing HST run from Ashley et al. (2020) (labelled 0).

\begin{tabular}{|c|c|c|c|}
\hline Run number & $T_{0}(\mathrm{BMJD}$ TDB) & $\sigma_{T 0}$ & Cycle \\
\hline 0 & 57814.9463288 & 0.0000070 & -2383862 \\
\hline 0 & 57815.0043813 & 0.0000059 & -2383733 \\
\hline 0 & 57815.0835622 & 0.0000052 & -2383558 \\
\hline 1 & 58919.9207031 & 0.0000128 & 70872 \\
\hline 2 & 58921.8984927 & 0.0002144 & 75265 \\
\hline 3 & 58922.9163339 & 0.0002217 & 77526 \\
\hline 4 & 58923.8926391 & 0.0005235 & 79695 \\
\hline 5 & 58931.9447283 & 0.0000133 & 97583 \\
\hline 6 & 58971.1515796 & 0.0000113 & 184682 \\
\hline 7 & 58999.0953122 & 0.0004916 & 246760 \\
\hline 8 & 59061.0036053 & 0.0000085 & 384292 \\
\hline 9 & 59061.1925888 & 0.0000093 & 384711 \\
\hline 9 & 59061.2038316 & 0.0000071 & 384736 \\
\hline 10 & 59243.2513696 & 0.0005262 & 789161 \\
\hline 11 & 59259.2425308 & 0.0000252 & 824685 \\
\hline 12 & 59259.2465427 & 0.0000125 & 824694 \\
\hline 13 & 59262.1911550 & 0.0000088 & 831236 \\
\hline 14 & 59273.9344339 & 0.0000152 & 857324 \\
\hline 15 & 59274.9407973 & 0.0000382 & 859560 \\
\hline 16 & 59275.9355367 & 0.0000145 & 861770 \\
\hline 17 & 59276.1437909 & 0.0005388 & 862232 \\
\hline 18 & 59282.9359955 & 0.0000166 & 877321 \\
\hline 19 & 59291.1902899 & 0.0002677 & 895658 \\
\hline 20 & 59293.8860530 & 0.0000110 & 901647 \\
\hline 21 & 59293.9418275 & 0.0000150 & 901771 \\
\hline 22 & 59304.9117311 & 0.0000745 & 926141 \\
\hline 23 & 59305.9190805 & 0.0000258 & 928379 \\
\hline 24 & 59306.2321825 & 0.0012266 & 929074 \\
\hline 25 & 59313.8161005 & 0.0000109 & 945922 \\
\hline 25 & 59313.9096758 & 0.0000160 & 946130 \\
\hline 26 & 59314.8774209 & 0.0000443 & 948280 \\
\hline 27 & 59322.8937543 & 0.0000143 & 966089 \\
\hline 28 & 59323.8065797 & 0.0000135 & 968117 \\
\hline 28 & 59323.9149702 & 0.0000162 & 968357 \\
\hline 29 & 59335.1223806 & 0.0001813 & 993255 \\
\hline 30 & 59336.8887346 & 0.0000121 & 997179 \\
\hline 31 & 59337.8330519 & 0.0000144 & 999277 \\
\hline 31 & 59337.8947119 & 0.0000199 & 999414 \\
\hline 32 & 59338.8534525 & 0.0000594 & 1001544 \\
\hline 33 & 59339.8801360 & 0.0000240 & 1003824 \\
\hline 34 & 59353.9416886 & 0.0000122 & 1035063 \\
\hline 34 & 59353.9691594 & 0.0000121 & 1035124 \\
\hline 34 & 59354.0204276 & 0.0000122 & 1035238 \\
\hline 34 & 59354.0487706 & 0.0000117 & 1035300 \\
\hline 34 & 59354.0613222 & 0.0000122 & 1035328 \\
\hline 34 & 59354.0689996 & 0.0000111 & 1035345 \\
\hline 34 & 59354.0847275 & 0.0000119 & 1035380 \\
\hline 34 & 59354.1009802 & 0.0000117 & 1035416 \\
\hline 34 & 59354.1135250 & 0.0000120 & 1035444 \\
\hline 34 & 59354.1441434 & 0.0000120 & 1035512 \\
\hline 34 & 59354.2134852 & 0.0000123 & 1035666 \\
\hline 35 & 59355.1316792 & 0.0000091 & 1037706 \\
\hline 35 & 59355.2036616 & 0.0000108 & 1037866 \\
\hline
\end{tabular}

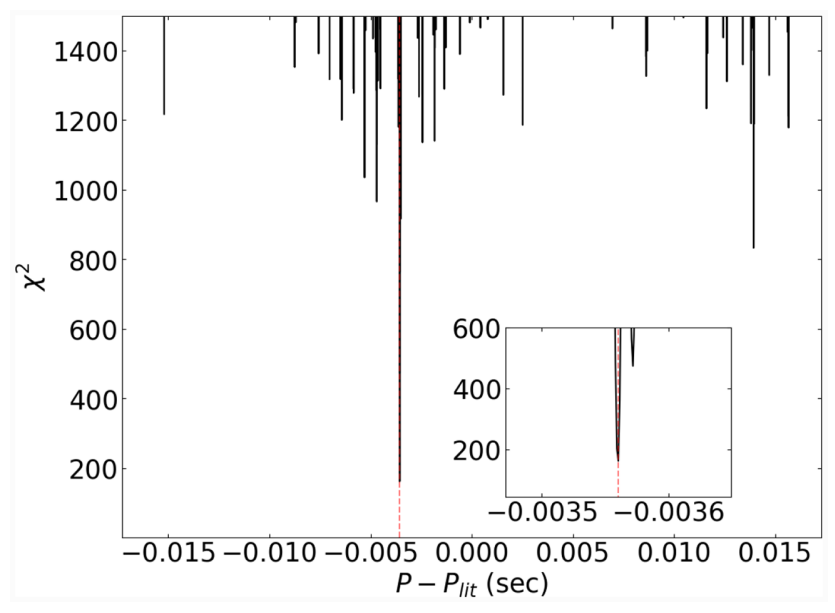

Figure 3. Minimum value of $\chi^{2}$ of a linear ephemeris fit over a range of periods spanning five times the uncertainty reported in Ashley et al. (2020). The period range is shown in terms of the difference to the period in Ashley et al. (2020), $P_{l i t}$. Our best period is indicated by the vertical dashed line. The inset shows a zoom around the best period, showing that there are no competing period aliases with similar $\chi^{2}$ values.

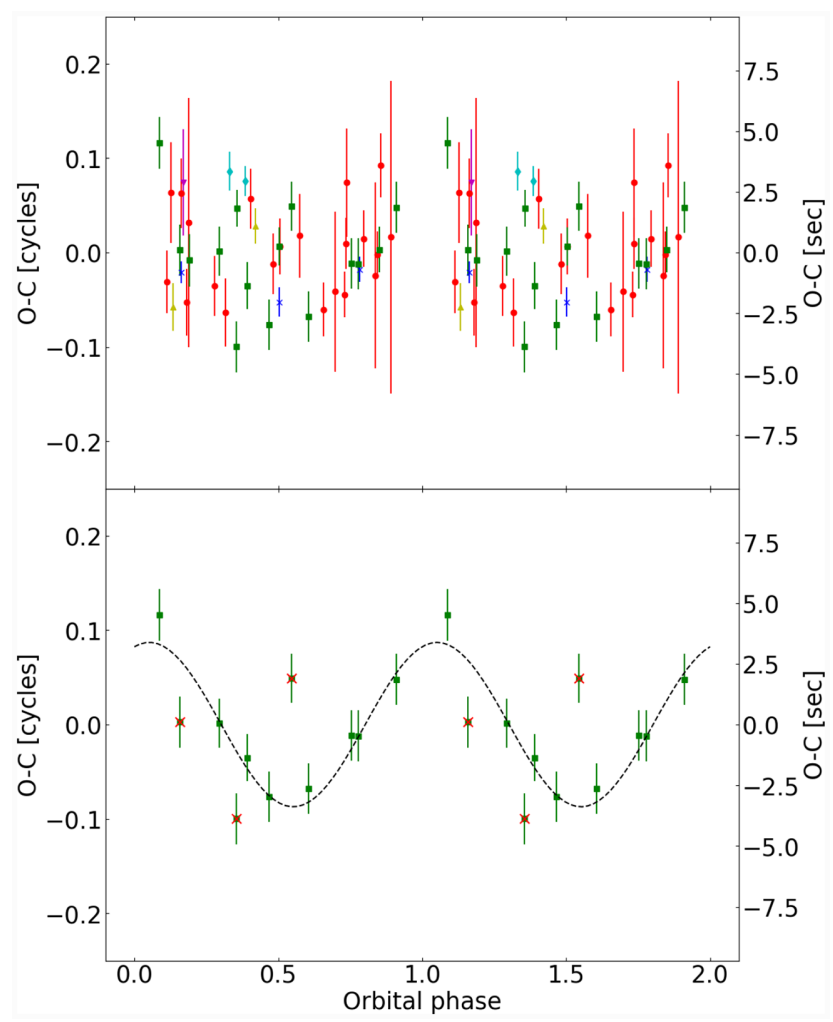

Figure 4. Top panel: pulse timing delay as a function of the orbital phase. Ashley et al.'s (2020) HST data are shown as blue crosses, TNT data as red circles, WHT data as green squares, LT data as magenta up-sidedown triangles, NOT data as yellow triangles, and CHIMERA data as cyan diamonds. Points with uncertainty larger than 30 per cent of the spin period are not shown. A hint of orbital modulation can be noticed, with pulses presenting a slight delay around phases 0 and 1.0, and a shorter arrival time around phase 0.5 . Bottom panel: same as the top panel, but showing only the continuous 7.3-h WHT run. Removing the effect of instrument sensitivity and minimizing weather effects makes the orbital modulation clear. The dashed line shows a least-squares fit to model this modulation and calculate a correction. Points marked by crosses were not used in the fit. 
To minimize the effect of observing conditions and remove the effect of different instruments, we have repeated the same exercise for a single WHT run with a duration of $7.3 \mathrm{~h}$. Given the high quality of the WHT data, the spin period can often be detected within a short-time interval. To optimize the number of detections for this run, our procedure was to start with a small chunk of data spanning $5 \mathrm{~min}$, and compute the value of $T_{0}$ as described in Section 3.2. If the obtained value had an uncertainty smaller than 3 per cent of the spin period, it was accepted; otherwise, the time interval was increased by $2 \mathrm{~min}$. This was repeated iteratively until the desired uncertainty was achieved, or the time interval reached an hour. Once a value was accepted, the same procedure was repeated for the next chunk of data, starting with the first value after the previous time interval, i.e. we allowed for no overlap between the data ranges used for each detection. The result is shown in the bottom panel of Fig. 4. With this more homogeneous data set, the orbital modulation becomes clear.

We can therefore estimate a correction to derived $T_{0}$ values depending on the orbital phase in order to remove any effects of modulation. This modulation is not solely the result of the white dwarf orbital motion, which can explain at most $\sim 1.8 \mathrm{~s}$ out of the observed range of $\sim 7 \mathrm{~s}$, but is likely largely caused by the effect of beat signals slightly shifting the period peak. As shown in Fig. 4, we calculate the modulation correction by fitting a sinusoidal curve to the WHT data. We obtained the following equation:

$T_{0 \text { corr }}=3.40(43) \mathrm{s} \times \sin \left[2 \pi \varphi_{\text {orb }}+1.25(6)\right]$,

where $\varphi_{\text {orb }}$ is the orbital phase of each measurement, and the phase constant shown is in radians. Uncertainties were obtained by bootstrapping. We note that this correction is not applied for the times shown in Table 2. Applying this correction, we have rederived our ephemeris, obtaining the following equation:

$\mathrm{BMJD}(\mathrm{TDB})=58888.0183511(17)+0.0004498988053(12) \mathrm{E}$

Similarly, we can also investigate any orbital modulation of the spin amplitude. Fig. 5 shows the estimated pulse amplitude as a function of orbital phase. Unlike the pulse times, the amplitudes show no dependence on the orbital phase, and the observed scatter is consistent with a Gaussian distribution according to a normality test.

\subsection{The long-term spin behaviour of the white dwarf}

With a baseline of only just over $4 \mathrm{yr}$, and with essentially just three observing 'seasons' in place, it is too early to constrain the spin evolution of the white dwarf in V1460 Her. However, already we have some sensitivity to the short evolutionary time-scales seen in the systems AE Aqr and AR Sco discussed in the introduction.

After applying the correction given by equation (2) to our derived pulse times, we have inspected the $\mathrm{O}-\mathrm{C}$ diagram as a function of cycle number as shown in Fig. 6. We show for comparison the expected $\mathrm{O}-\mathrm{C}$ behaviour for a spin-down of $P / \dot{P}=5 \times 10^{6} \mathrm{yr}$ (as estimated for AR Sco, Stiller et al. 2018), and for $10^{7} \mathrm{yr}$ (that of AE Aqr, de Jager et al. 1994). From our current data set, there does not seem to be any indication of spin variability for V1460 Her, at least in time-scales comparable to the these two similar systems. The $\mathrm{O}-\mathrm{C}$ measurements are instead scattered around 0 (see Fig. 6).

In order to place a lower limit on the evolutionary time-scale of the spin period, we performed a quadratic fit to the ephemeris, corrected for the orbital modulation, using the Markov chain Monte Carlo method, implemented with EMCEE (Foreman-Mackey et al. 2013). As expected, the quadratic term is poorly constrained and consistent with 0 . Adopting the 1 per cent percentile as the lower limit, we obtain

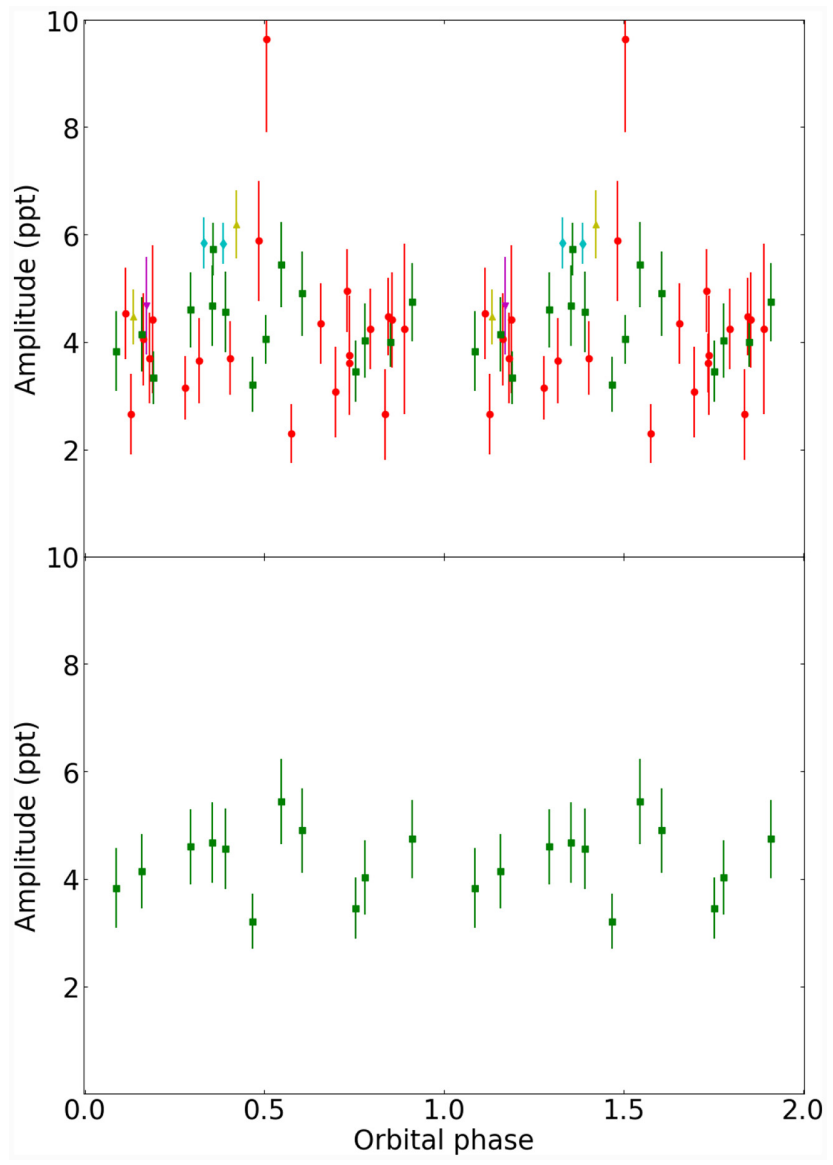

Figure 5. Top panel: amplitude of the spin pulsation as a function of the orbital phase. Symbols are the same as in Fig. 4. There is no evidence of amplitude variability, and the scatter is consistent with Gaussian noise. Bottom panel: same as the top panel, for only the continuous 7.3-h WHT run.

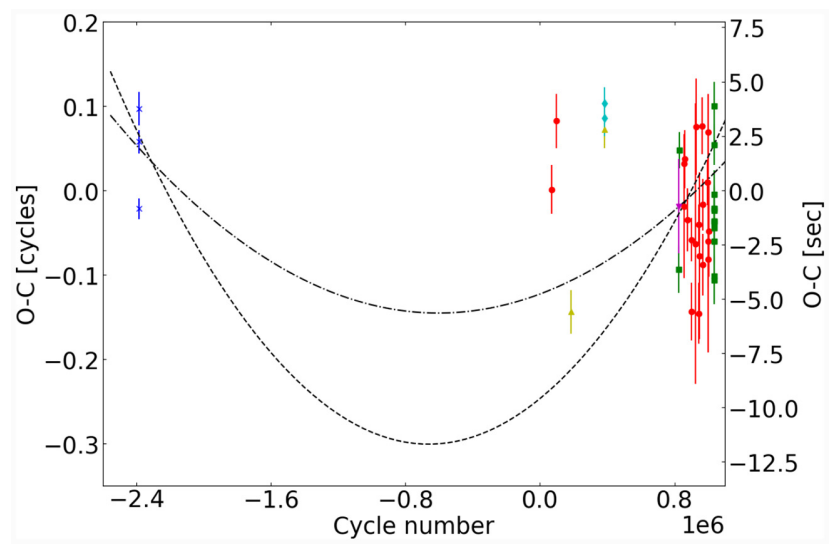

Figure 6. Pulse timing delay as a function of time. Symbols are the same as for Fig. 4. The dashed line shows a best fit to the data assuming the spin-down time-scale of AR Sco $\left(P / \dot{P}=5 \times 10^{6} \mathrm{yr}\right)$, and the dot-dashed line shows the same for $\operatorname{AE} \operatorname{Aqr}\left(P / \dot{P}=1 \times 10^{7} \mathrm{yr}\right)$. Although sparsely sampled in the first $3 \mathrm{yr}$ of coverage, the measurements around cycle number 0 suggest that V1460 Her does not have spin variations of comparable magnitude. 
$|P / \dot{P}|>4 \times 10^{7}$ yr. With the detection of the optical pulsations and an ephemeris secure over a time-scale of years, it should be possible to improve upon this limit rapidly in the future since any quadratic signal grows with the square of the baseline. However, this timescale, which is longer than is seen in either AE Aqr or AR Sco, suggests that V1460 Her may be in a state of quasi-equilibrium as opposed to a short-lived state of spin-down.

\section{SUMMARY AND CONCLUSIONS}

We have detected the spin pulsations of V1460 Her in the optical for the first time. This is particularly important for long-term monitoring of the spin, as optical detection implies that observations can be carried out with ground-based telescopes. Our data show that the spin can be detected even for 2-m class telescopes like the TNT, provided that the observing conditions are favourable.

We have executed 35 observing runs over more than 1 yr. Combining our observations with the HST data from Ashley et al. (2020), we obtained a total of 44 detections of the spin with timing uncertainty better than 30 per cent, given that some runs allowed for more than one measurement. This allowed for a much more precise estimate of the ephemeris (equation 1) compared to the values of Ashley et al. (2020).

We have also identified an orbital modulation of the pulse arrival times. Modelling this effect, we have derived a correction (equation 2), which we applied to our derived times to present improved ephemeris (equation 3), and to investigate the long-term behaviour of the spin frequency. Although we believe we see signs of modulation of the pulse phase with orbital period, we note that the amplitude of the correction is less than 0.1 spin cycles, so our conclusions are not qualitatively affected even if we apply no correction.

With the current baseline of just over $4 \mathrm{yr}$, albeit sparsely sampled at the start, we find no evidence for any change in the spin period of V1460 Her, and place a lower limit on the time-scale of spin period changes of $|P / \dot{P}|>4 \times 10^{7} \mathrm{yr}$. This suggests that V1460 Her could currently be in a state of quasi-equilibrium caused by a balance between accretion spin-up, and spin-down triggered by magnetic torque. Measuring the spin variability in accreting fast-spinning white dwarfs can ultimately enable us to probe the origin of magnetic fields in cataclysmic variables (Schreiber et al. 2021), providing constraints on the evolution of interacting compact binaries. Therefore, following the main result of this work that the spin pulsations can be detected in the optical, we encourage continuous monitoring of this system in order to confirm the quasiequilibrium behaviour, or possibly measure the spin change with a longer baseline than presented here.

\section{ACKNOWLEDGEMENTS}

We thank the referee John Thorstensen for reviewing our manuscript. IP and TRM acknowledge support from the UK's Science and Technology Facilities Council (STFC), grant ST/T000406/1, and from the Leverhulme Trust. AA acknowledges support from Thailand Science Research and Innovation (TSRI) grant FRB640025 contract no. R2564B006. VSD is supported by the STFC grant ST/V000853/1. SGP acknowledges the support of a STFC Ernest Rutherford Fellowship.

This work has made use of data obtained at the Thai National Observatory on Doi Inthanon, operated by NARIT. The data presented here were partially obtained with ALFOSC, which is provided by the Instituto de Astrofisica de Andalucia (IAA) under a joint agreement with the University of Copenhagen and NOT.

\section{DATA AVAILABILITY}

All data analysed in this work can be made available upon reasonable request to the authors.

\section{REFERENCES}

Ashley R. P. et al., 2020, MNRAS, 499, 149

Belokurov V. et al., 2020, MNRAS, 496, 1922

Bookbinder J. A., Lamb D. Q., 1987, ApJ, 323, L131

Campbell C. G., 1983, MNRAS, 205, 1031

de Jager O. C., Meintjes P. J., O’Donoghue D., Robinson E. L., 1994, MNRAS, 267, 577

Dhillon V. S. et al., 2014, MNRAS, 444, 4009

Foreman-Mackey D., Hogg D. W., Lang D., Goodman J., 2013, Publ. Astron. Soc. Pac., 125, 306

Fuller J., Lai D., 2012, MNRAS, 421, 426

Gaibor Y., Garnavich P. M., Littlefield C., Potter S. B., Buckley D. A. H., 2020, MNRAS, 496, 4849

Garnavich P., Littlefield C., Lyutikov M., Barkov M., 2021a, ApJ, 908, 195

Garnavich P., Littlefield C., Wagner R. M., van Roestel J., Jaodand A. D., Szkody P., Thorstensen J. R., 2021b, ApJ, 917, 22

Harding L. K. et al., 2016, MNRAS, 457, 3036

Ikhsanov N. R., 1998, A\&A, 338, 521

Isern J., García-Berro E., Külebi B., Lorén-Aguilar P., 2017, ApJ, 836, L28

Israel G. L., Stella L., Angelini L., White N. E., Kallman T. R., Giommi P., Treves A., 1997, ApJ, 474, L53

Joss P. C., Katz J. I., Rappaport S., 1979, ApJ, 230, 176

Katz J. I., 2017, ApJ, 835, 150

Kennedy M. R., Garnavich P., Breedt E., Marsh T. R., Gänsicke B. T., Steeghs D., Szkody P., Dai Z., 2016, MNRAS, 459, 3622

Liebert J. et al., 2005, AJ, 129, 2376

Liebert J., Ferrario L., Wickramasinghe D. T., Smith P. S., 2015, ApJ, 804, 93

Littlefield C., Garnavich P., Kennedy M., Callanan P., Shappee B., Holoien T., 2017, ApJ, 845, L7

Littlefield C. et al., 2020, ApJ, 896, 116

Livio M., Pringle J. E., 1998, ApJ, 505, 339

Lopes de Oliveira R., Bruch A., Rodrigues C. V., Oliveira A. S., Mukai K., 2020, ApJ, 898, L40

Lyutikov M., Barkov M., Route M., Balsara D., Garnavich P., Littlefield C., 2020, preprint (arXiv:2004.11474)

Marsh T. R. et al., 2016, Nature, 537, 374

Mereghetti S., La Palombara N., Tiengo A., Pizzolato F., Esposito P., Woudt P. A., Israel G. L., Stella L., 2011, ApJ, 737, 51

Morales-Rueda L., Maxted P. F. L., Marsh T. R., North R. C., Heber U., 2003, MNRAS, 338, 752

Paczynski B., Zytkow A. N., 1978, ApJ, 222, 604

Pala A. F. et al., 2020, MNRAS, 494, 3799

Parsons S. G., Gänsicke B. T., Schreiber M. R., Marsh T. R., Ashley R. P., Breedt E., Littlefair S. P., Meusinger H., 2021, MNRAS, 502, 4305

Patterson J., 1979, ApJ, 234, 978

Patterson J., 1984, ApJS, 54, 443

Patterson J., 1994, Publ. Astron. Soc. Pac., 106, 209

Patterson J. et al., 2020, ApJ, 897, 70

Schenker K., King A. R., Kolb U., Wynn G. A., Zhang Z., 2002, MNRAS, 337, 1105

Schreiber M. R., Belloni D., Gänsicke B. T., Parsons S. G., Zorotovic M., 2021, Nat. Astron., 5, 648

Stiller R. A., Littlefield C., Garnavich P., Wood C., Hambsch F.-J., Myers G., 2018, AJ, 156, 150

Thorstensen J. R., 2020, AJ, 160, 151

Wolf W. M., Bildsten L., Brooks J., Paxton B., 2013, ApJ, 777, 136

Wynn G. A., King A. R., Horne K., 1997, MNRAS, 286, 436

This paper has been typeset from a $\mathrm{T}_{\mathrm{E}} \mathrm{X} / \mathrm{LAT} \mathrm{E}$ file prepared by the author. 